\title{
The Effect of Lending Structure Concentration on Credit Risk: The Evidence of Vietnamese Commercial Banks
}

\author{
Thi Thu Diem LE ${ }^{1}$, Thanh Tung DIEP ${ }^{2}$
}

Received: April 09, 2020 Revised: May 17, 2020 Accepted: June 07, 2020

\begin{abstract}
This paper examines whether lending structure can lower credit risk by employing econometric techniques of panel data for the Vietnamese banking system at the bank level used by economic sectors from 2011 to 2016 . New light is being shed on assessing the impact of each industry's debt outstanding on credit risk. Adopting findings from previous studies, we assess credit risk from two different sources, including loan loss provision and non-performing loan. Moreover, we also focus on observing lending structure in many different aspects, from concentrative levels to the short-term and long-term stability levels of lending structure. The Generalized Method of Moments (GMM) estimator was applied to analyze the relationship between concentration and banking risks. In general, the results show that lending concentration may decrease credit risk. It is interesting to observe that the Vietnamese commercial bank lending portfolios have, on average, higher levels of diversity across different sectors. In particular, the increase in hotel and restaurant lending contributes to decrease credit risk while the lending portfolios of banks in agriculture, electricity, gas and water increase credit risk. This study suggests the need for further analysis and research about portfolio risks in lending activities for maintaining efficiency and stability in the commercial banking system.
\end{abstract}

Keywords : Financial Economics, Lending Structure, Bank Risks, Credit Risks, Commercial Banks.

JEL Classification Code: G21, G23, G32, H21

\section{Introduction}

The subject of bank risks is an important issue that was re-emphasized during the financial crisis of 2007. It forces banking systems to face major challenges in the form of increased bank risks, concentration, and restructuring. Therefore, examining the sources of bank risks (Tran \& Nguyen, 2020) is essential for bank regulators and investors. Many notable exposures have triggered a lot of research to investigate the lending structure to reduce bank risks. Importantly, most researchers have put the focus on developed markets (Bassett, Demiralp, \& Lloyd, 2020; Rossi,

${ }^{1}$ First Author and Corresponding Author. Deputy Dean, School of Economics and Law, Tra Vinh University, Vietnam [Postal Address: No. 126, Nguyen Thien Thanh, Hamlet 4, Ward 5, Tra Vinh City, Tra Vinh Province, 940000, Vietnam] Email: Ittdiem@tvu.edu.vn ${ }^{2}$ Dean, School of Economics and Law, Tra Vinh University, Vietnam. Email: dttung@tvu.edu.vn

() Copyright: The Author(s)

This is an Open Access article distributed under the terms of the Creative Commons Attribution Non-Commercial License (http://Creativecommons.org/licenses/by-nc/4.0/) which permits unrestricted noncommercial use, distribution, and reproduction in any medium, provided the original work is properly cited.
Schwaiger, \& Winkler, 2009; van der Veer \& Hoeberichts, 2016) where banks are at a mature development stage or in Islamic countries (Abdul-Rahman, Sulaiman, \& Mohd Said, 2018; Rahman, 2010) where banks are operating in a different type of markets. In contracts, banks in emerging markets are currently at an earlier stage of development and bank risk is not a priority.

Similar to many emerging markets, the Vietnamese equity market is small and fragile to even minor shocks (Vo, 2015). Because of this, Vietnam is considered a bank-based economy where most of the firm financing is from bank credit (Vo, 2017). In other words, loans are the most important types of assets that banks hold. In these conditions, Vietnamese banking specialists claim that credit has been growing, reaching nearly 20 percent in the last three years (Vietnamnet, 2017). However, according to data published by the State Bank Vietnam, Vietnamese market banks continue to grapple with growing credit exposure, reporting that credit exposure in the banking system was only $3.5 \%$ (USD4 billion) as of 30 June 2011. Bank exposure due to the problematic real-estate sector and securities sector accounted for $12 \%$ (USD12 billion) of the total loan book of the banking system. High sector concentration in lending 
portfolios arises from excessive exposure to a single sector or several highly correlated sectors. Some international institutions such as the World Bank and the International Monetary Fund are concerned that rapid bank loans with high sector concentration in Vietnam would bring high risks to the economy. This issue highlights the high level of risks in Vietnamese bank portfolios in the context of increased risktaking by banks.

This paper contributes to the literature on several fronts. Firstly, it extends the current literature by providing more explanation for the issue of credit risk by critically reviewing previous studies (Onyiriuba, 2016; Rahman, 2010; Rossi et al., 2009; Tabak, Fazio, \& Cajueiro, 2011). Also, a large set of risks are taken into consideration while being compared with those by Rahman (2010), Acharya, Hasan, and Saunders (2006) and Chen, Wei, and Zhang (2013). Besides, more robustness and reliable results in the relationship between lending structure concentration and credit risk can be produced with the use of different measures explaining credit risk. Finally, yet importantly, the number of indicators representing concentration was used to explore lending structures. The specialization index in the paper, similarly to the HerfindahlHirschman Index, is the most utilized concentration measure in the field of industrial organization. Especially, in this study, lending composition change index and variance of traditionality index were used, allowing for the examination of short-term and medium-term stabilities in the lending structure. More importantly, the effect of sector concentration in loan portfolios on credit risk was also highlighted. Therefore, it can be stated that there is a positive correlation between concentration measures and reliability of the results.

In the current bank risk-related literature, the relationship between concentration and credit risk has been theoretically highlighted. For example, concentration in economic sectors decreases credit risk and enhances banking efficiency (Rossi et al., 2009). Meanwhile, the impact of lending structure concentration in economic sectors on the bank risk in Malaysian commercial banks in the short term, medium term and long term is carefully noted by Rahman (2010). In addition, Tabak et al. (2011) suggested that lending structure concentration in economic sectors has contributed to the increase in profits and the reduction in credit risk for the Brazilian commercial banking system. Moreover, the influential intensity negatively affected banks' focus on lending structures by scale or in the form of ownership, especially after the world financial crisis. Therefore, the impact of lending structure concentration on credit risk, considering the short-term, as well as long-term impacts on commercial banks, needs to be assessed. However, these issues have not been addressed to any extent in the context of the Vietnamese banking system. In addition, the consideration of specific impacts of economic sectors on credit risk needs to identify the sectors on which banks should place more focus, underlying the important role in developing banks' business strategies. Therefore, this study is expected to provide empirical evidence on the impact of a lending strategy on bank risks.

\section{Literature Review}

\subsection{Lending Structure Concentration}

The traditional banking theory suggests that banks should diversify their loan portfolios in order to reduce credit risks. This theoretical suggestion also corresponds to the portfolio theory by Markowitz (1959). Grounded on the theory of asymmetric information, Diamond (1984) states that diversification allows banks to transform monitored debt into unmonitored debt because every concentrated bank would be more vulnerable to economic downturns when they expose themselves to few sectors. Moreover, the increased diversification efforts in terms of industry and sizes of the borrowing companies lead to lowering future provisions, which could result in a reduction of realized risks (Berger \& DeYoung, 1997; Rossi et al., 2009).

Furthermore, the view that firms concentrate their activities in a specialized sector for comparative benefits because diversification can result in increasing competition, making diversification strategies less attractive, is supported by the theory of corporate finance (Acharya et al., 2006; Behr, Kamp, Memmel, \& Pfingsten, 2007; Kahn \& Winton, 2004; Tabak et al., 2011). Acharya et al. (2006) find that, for a high-risk bank, loan expansion to new industries usually results in riskier loans. By using the seasonal data of borrowers for determining the degree of diversification in bank credit portfolios, Behr et al. (2007) empirically support this view, stating that specialized banks have lower relative loan loss provisions and lower shares of non-performing loans. More recently, Silva, Alexandre, and Tabak (2018) highlight empirical evidence supporting the claim that more diversified portfolios of banks would contribute to higher sector risk levels. In the same vein, in Vietnam, Batten and Vo (2015) note that bank diversification may also be associated with higher risks. Although a few investigations have been conducted into banks in Vietnam, this effect should need to be more intensively examined.

\subsection{Credit Risks}

Policymakers and supervisors who are responsible for maintaining financial stability focus on credit risks (Haq \& Heaney, 2012; Stiroh, 2004). It is also assumed that bank concentration has a negative effect on growth in financially dependent industries (Diallo, 2017). By examining the effect of lending structure concentration on credit risks (Acharya et al., 2006; Tabak et al., 2011) the researchers show evidence 
that non-performing loans can be reduced by loan portfolio concentration. In addition, Acharya et al. (2006) point out two reasons to explain why an increase in diversification might also raise the risk of bank loan portfolios. The first reason is that banks may suffer from lower monitoring efficiency if they expose their loans to new sectors. The second reason is that diversification can lead to increasing a bank's scope, subjecting it to scale inefficiencies.

In Vietnam, the number of studies have focused on diversification (Batten \& Vo, 2016; Vo, 2017). For example, Batten and Vo (2016) investigated risk shifting in commercial banks in the emerging market where banks fund domestic asset portfolios almost exclusively from deposits and with the limited issuance of securities. The findings support the importance of linking deregulation with financial market openness and transparency to enhance and stimulate international portfolio investments. In additions, Vo (2017) investigated how the stock market values bank diversification within the social context, stating that there is a negative relationship between bank diversification and stock market valuation. However, there are no published findings on lending structure concentration and bank risks, which is specific to Vietnam's banking settings.

\subsection{The Relationship between Lending Structure Concentration and Credit Risks}

The number of studies suggests that concentration not only reduces risks, but also decreases the greater risk for banks. For example, Acharya et al. (2006) find that diversification does not bring superior and safer performance for banks, whereas concentration takes advantages of favorable conditions in one existing industry instead of new industries. Tabak et al. (2011) also find that a focus on credit portfolios increases bank efficiency and reduces loss rates. However, Rahman (2010) argues that concentration into the number of industries such as real-estate in the portfolio may lead to an increase in loan losses dues to the effects of banking risks. Showing the same tendency, Blasko and Sinkey (2006) suggest that lending structure concentration in the real-estate industry can lead to many difficulties in managing interest rate risks. In addition, Diallo (2017) provides evidence that lending structure concentration has an adverse impact on the development of the financial-dependent industry. Overall, these studies show that diversification is significantly related to banking risks both positively and negatively. In this study, the positive effect of lending structure concentration is expected on the banking risks.

H1: There exists a negative relationship between concentration of lending structure and the credit risks.

Moreover, Onyiriuba (2016) classifies an asset by its maturity. Therefore, strategies to diversify bank credit portfolios need to be considered not only in short terms, but also in a longer vision. Unsurprisingly, the State Bank of Vietnam adjusts its monetary policy. For example, when the State Bank lowers lending rates for some governmentoriented industries, commercial banks can obtain lower profits than those in other sectors. Consequently, some banks tend to lower credit standards for some industries causing the lending structure to be riskier in the future. Therefore, maintaining the strategy of lending structure concentration over the medium and long-term can make banks more vulnerable to unpredictable macroeconomic fluctuations.

H2: There exists a positive relationship between shortterm stability in lending composition and credit risks.

H3: There exists a positive relationship between lending composition change in the variance of traditionality in the medium and/or long term and credit risks.

\section{Model and Estimation Methods}

\subsection{Model}

The economic approach is similar to those of AbdulRahman et al. (2018), Chen et al. (2013), Tabak et al. (2011), Luong, Nguyen, and Nguyen (2020). An estimation which consists of the Generalized Method of Moments (GMM) estimators is proposed as in the following equation

$$
\begin{aligned}
& \mathrm{Y}= \mathrm{b} 0+\mathrm{b} 1 \mathrm{X}+\mathrm{b} 2 \mathrm{TL}+\mathrm{b} 3 \mathrm{TE}+\mathrm{b} 4 \mathrm{INV} \\
& \mathrm{b} 6 \mathrm{NONII}+\mathrm{b} 7 \mathrm{GDP}+\mathrm{b} \text { INTEXP }+\varepsilon,
\end{aligned}
$$

where Y represents credit risk with the four measures including two measures of credit risk, and $\mathrm{X}$ respectively is outstanding loans by economic sectors (RISKY1, RISKY2, RISKY3, RISKY4, RISKY5, RISKY6, RISKY7, RISKY8, RISKY9), Lending concentration (SPEC), Lending composition change (LCC) and Variance of traditionality index (VART).

$\varepsilon$ is the error term

\subsection{Variable Description}

\subsubsection{Dependent Variable - Credit Risk}

Following the approach of Ozili and Outa (2017), credit risks and the ratio of total loan loss provisions to total loan are identified at the end of the fiscal year. Besides, the ratio of loan loss provisions (LLP) to total loan is usually a measure of expected credit risk; the paper also considers other measures of unexpected credit risks measured as the standard deviation of non-performing loan ratio (STDNPL) (Acharya et al., 2006). 


\subsubsection{Independent Variables}

Depending on the matching of bank portfolio structure, and similar to Kildegaard and Williams (2002), Nepp, Lavysh, Kuprina, and Nikonov (2012) and Chen et al. (2013), in this study, nine industries were selected.

\section{Risky sector (RISKY)}

Attempts were made at studying the impact of sector concentration in loan portfolios on credit risk. Slightly different to Nepp et al. (2012), this study used nine lending measures such as wholesale and retail trade $\left(\right.$ RISKY $\left._{1}\right)$; agriculture (RISKY ${ }_{2}$ ); mining and quarrying (RISKY ${ }_{3}$ ); manufacturing $\left(\right.$ RISKY $\left._{4}\right)$; electricity, gas and water $\left(\right.$ RISKY $\left._{5}\right)$; transport and communications (RISKY $)_{6}$; construction and real estate $\left(\right.$ RISKY $\left._{7}\right)$; hotels and restaurant $\left(\right.$ RISKY $\left._{8}\right)$; other community, social and personal services (RISKY,

\section{Lending concentration (SPEC)}

Similar to Berger, Minnis, and Sutherland (2017) and Dao and Nguyen (2020), SPEC is constructed as in this equation

$$
\operatorname{SPEC}_{i, t}=\sum_{i=1}^{9} S_{j i t}^{2}
$$

where $\mathrm{Sj}, \mathrm{i}, \mathrm{t}$ is the amount of annual lending of sector $\mathrm{j}$ of bank $i$ in year t. A score approaching 1 indicates the high level of lending concentration while a score approaching 0 indicates a high level of diversity in loan portfolios across different sectors.

Lending composition change (LCC)

LCC refers to the short-term stability in the lending composition. LCC is generated by using the equation

$$
L C C_{i, t}=\sum_{j=1}^{9} \min \left(S_{j, i, t}, S_{j, i, t-1}\right)
$$

where $S_{j, i,}$ and $S_{j, i, t-1}$ are the contribution to the amount of annual lending of bank $i$ industry $j$ in year $t$ and $t-1$. It takes a maximum value of 1 if there is no change in the composition of the lending and the minimum value of 0 if the portfolio of lending through financial sectors is not given in the previous year. Therefore, a high LCC value indicates the short-term stability of the lending composition.

\section{Variance of traditionality index (VART)}

VART represents the stability of the medium-term lending structure. It is the variance of traditionality index (TI) that is calculated by using three-year intervals for each of the sectors involved. TI for 2011 is calculated using the data from 2010 to 2012, while TI for 2012 uses the 2011-2013 data. In order to optimize observations with data particularly, this study chose three years intervals for calculating TI. The formula for TI is as in this equation

$$
T I_{j, i, t}=\frac{\sum_{t=t-1}^{t=t+1} C_{j, i, t}}{3}
$$

where accumulated lending for each industry, $\mathrm{C}_{\mathrm{i}, \mathrm{j}, \mathrm{t}}$, is constructed as the following equation

$$
C_{j, i, t}=\frac{e_{j, i, t}}{\sum_{t=t_{0}}^{t_{1}} e_{j, i, t}}
$$

where $t_{0}$ and $t_{1}$ are the beginning and end of the period for the data, and ej,i,t is lending sector $\mathrm{j}$ of bank $\mathrm{i}$ in year t. VART is the variance of the entire TI, showing different lending patterns in the following 3 years.

\subsubsection{Control Variables}

Our control variables were selected based on the bankspecific effects. As suggested by the literature, credit risk is driven by certain bank characteristics identified. Among those, LTA is the logarithm of the bank total assets at the end of the fiscal year. TL is the ratio of total loan to total asset at the end of the fiscal year. TE measures the financial strength, calculated as total equity divided by total asset. INTEXP and NONII are the ratios of income structures evaluating the effect of non-interest and interest activities on the tradeoff in a bank's profitability and risk, calculated as interest expenses divided by total asset and noninterest expense divided by total asset at the year end respectively. INV is the proportion of investment except for bank loans to total asset. In the context of high competition, bank investment plays an important role in increasing bank profitability because banks generate more profits through finance diversity.

With regards to macroeconomic factor, the GDP is measured by the growth of Gross Domestic Product. GDP is commonly used as an indicator of the economic health of a country and this variable may be a key proxy of bank studies to measure the demand for banking services such as deposits and loans.

\subsection{Data}

Ten traded commercial banks in Vietnam over the period from 2011 to 2016, listed in the Ho Chi Minh City and Hanoi stock exchanges were first examined. For measuring VART, data of two additional years (2010 and 2017) for sector loans from financial statements were obtained because several variables used in our analyses were calculated by three-year intervals.

\section{Empirical Results}

The descriptive statistics of our sample are presented in Table 1. In particular, the number of observations, mean, 
standard deviation, and minimum, maximum for two samples with dependent and independent variables a year from 2011 to 2016 is reported in Table 1. The descriptive statistics were conducted to examine the statistical characteristic of each variable in the model. Mean value refers to the average value of the variables for the entire sample.

Before conducting panel regression estimations, correlation analysis was run to ensure the data are free from severe multicollinearity issue. Table 2 shows the correlation matrix between the dependent variables and independent variables. In general, the coefficient correlations for all variables are $<0.8$, conjecturing that multicollinearity problem is not severe for the data sets.

The GMM estimation (Arellano \& Bover, 1995) was applied to analyze the relationship between concentration and banking risks of eight Vietnamese commercial banks in the period from 2011 to 2016 . The estimations have passed the Sargan and Hassan tests on the limit of overdetermination and accepted the hypothesis of $\mathrm{HO}$ in the Arellano-Bond test of the autocorrelation phenomenon. Therefore, the estimated results are reliable (sees Table 3 and Table 4).

Table 1: Summary statistics of all variables over the period 2011-2016

\begin{tabular}{|c|c|c|c|c|c|}
\hline Variable & Obs & Mean & Std. Dev. & Min & Max \\
\hline LLP & 48 & 0.0135 & 0.0047 & 0.0080 & 0.0254 \\
\hline STDNPL & 48 & 0.1155 & 0.2398 & 0.0050 & 0.7425 \\
\hline $\mathrm{RISKY}_{1}$ & 48 & 0.2041 & 0.0868 & 0.0027 & 0.3574 \\
\hline $\mathrm{RISKY}_{2}$ & 48 & 0.0616 & 0.0601 & 0.0000 & 0.2250 \\
\hline $\mathrm{RISKY}_{3}$ & 48 & 0.0276 & 0.0272 & 0.0000 & 0.0918 \\
\hline $\mathrm{RISKY}_{4}$ & 48 & 0.1904 & 0.1005 & 0.0194 & 0.3699 \\
\hline $\mathrm{RISKY}_{5}$ & 48 & 0.0414 & 0.0350 & 0.0000 & 0.1185 \\
\hline $\mathrm{RISKY}_{6}$ & 48 & 0.0379 & 0.0216 & 0.0089 & 0.1003 \\
\hline $\mathrm{RISKY}_{7}$ & 48 & 0.1442 & 0.0902 & 0.0430 & 0.3824 \\
\hline $\mathrm{RISKY}_{8}$ & 48 & 0.0143 & 0.0085 & 0.0006 & 0.0301 \\
\hline $\mathrm{RISKY}_{9}$ & 48 & 0.2784 & 0.1947 & 0.0356 & 0.7511 \\
\hline LCC & 48 & 0.9166 & 0.0629 & 0.6863 & 0.9824 \\
\hline SPEC & 48 & 0.2534 & 0.1045 & 0.1447 & 0.5798 \\
\hline VART & 48 & 0.0527 & 0.0362 & 0.0000 & 0.1291 \\
\hline $\mathrm{TL}$ & 48 & 0.5685 & 0.0977 & 0.3600 & 0.7000 \\
\hline TE & 48 & 0.0818 & 0.0213 & 0.0426 & 0.1476 \\
\hline INV & 48 & 0.1528 & 0.0504 & 0.0691 & 0.2769 \\
\hline LTA & 48 & 12.177 & 0.888 & 9.980 & 13.760 \\
\hline NONII & 48 & 0.0031 & 0.0025 & 0.0000 & 0.0086 \\
\hline GDP & 48 & 5.8683 & 0.5367 & 5.0300 & 6.6800 \\
\hline INTEXP & 48 & 0.0513 & 0.0176 & 0.0236 & 0.0873 \\
\hline
\end{tabular}

Note: Variable definitions STDNPL= the standard deviation of non-performing loan ratio, LLP $=$ the ratio of loan loss provisions to total loans, $\mathrm{RM}=$ market risk, $\mathrm{RSS}$ = idiosyncratic risk, $\mathrm{RISKY}_{1}$ (trade), $\mathrm{RISKY}_{2}$ (agriculture), $\mathrm{RISKY}_{3}$ (mining), $\mathrm{RISKY}_{4}$ (manufacturing), $\mathrm{RISKY}_{5}$ (electricity),

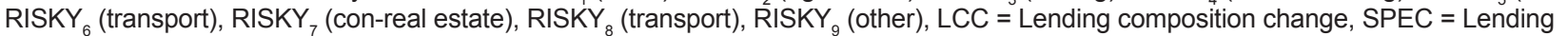
concentration, VART $=$ variance of traditionality index, TL $=$ total loan/total asset, TE $=$ total equity/total asset, INV $=$ investment/total asset, LTA $=$ logarithm of total assets, NONII = non-interest/total income, GDP = growth of Gross Domestic Product, INTEXP = interest/total income . 
Table 2: Correlation matrix for all banks

\begin{tabular}{|l|c|c|c|c|c|c|}
\hline & LLP & STDNPL & RISKY & RISKY $_{2}$ & RISKY $_{3}$ & RISKY $_{4}$ \\
\hline LLP & 1 & & & & & \\
\hline STDNPL & 0.2049 & 1 & & & & \\
\hline RISKY $_{1}$ & 0.3161 & 0.1688 & 1 & & & \\
\hline RISKY $_{2}$ & -0.1138 & -0.1087 & -0.2653 & 1 & & \\
\hline RISKY $_{3}$ & 0.4925 & 0.1544 & 0.3439 & 0.2642 & & \\
\hline RISKY $_{4}$ & 0.4630 & 0.0163 & 0.5326 & -0.1390 & 0.5884 & 1 \\
\hline RISKY $_{5}$ & 0.2042 & 0.4155 & 0.4632 & 0.0334 & 0.5022 & 0.3882 \\
\hline RISKY $_{6}$ & 0.2339 & 0.6306 & 0.0117 & -0.1295 & 0.4014 & 0.1728 \\
\hline RISKY $_{7}$ & -0.0434 & 0.0147 & -0.5401 & 0.3075 & -0.0828 & -0.3003 \\
\hline RISKY & -0.3656 & -0.3926 & 0.2534 & 0.0047 & 0.1298 & 0.0435 \\
\hline RISKY & -0.4405 & -0.2062 & -0.5324 & -0.2901 & -0.7810 & -0.7451 \\
\hline LCC & 0.0353 & -0.0763 & 0.2430 & 0.0172 & 0.0362 & 0.2157 \\
\hline SPEC & -0.2266 & -0.2813 & -0.5276 & -0.4516 & -0.6024 & -0.4884 \\
\hline VART & 0.0150 & -0.0328 & -0.3242 & -0.0930 & -0.3881 & -0.2325 \\
\hline TL & 0.3102 & -0.2957 & 0.1606 & 0.0038 & -0.0068 & 0.2912 \\
\hline TE & 0.0246 & 0.0870 & -0.3863 & -0.0989 & -0.2232 & -0.1641 \\
\hline INV & 0.0714 & 0.4752 & 0.0640 & -0.4111 & -0.0615 & 0.0033 \\
\hline LTA & 0.3307 & -0.0167 & 0.7742 & -0.1124 & 0.4366 & 0.7367 \\
\hline NONII & 0.2151 & 0.4891 & 0.6905 & -0.6105 & 0.1386 & 0.3758 \\
\hline GDP & -0.2752 & 0.0038 & 0.0522 & -0.0472 & -0.1655 & -0.1556 \\
\hline INTEXP & 0.2620 & -0.2392 & -0.2561 & 0.1520 & -0.0830 & -0.1313 \\
\hline Tab & & & & & \\
\hline
\end{tabular}

Table 2: Correlation matrix for all banks (next)

\begin{tabular}{|l|c|c|c|c|c|c|c|}
\hline & RISKY $_{6}$ & RISKY $_{7}$ & RISKY $_{8}$ & RISKY & LCC & SPEC & VART \\
\hline RISKY $_{6}$ & 1 & & & & & \\
\hline RISKY $_{7}$ & -0.0033 & 1 & & & & \\
\hline RISKY $_{8}$ & -0.0527 & -0.1949 & 1 & & & \\
\hline RISKY $_{9}$ & -0.2530 & -0.0948 & -0.1071 & 1 & & \\
\hline LCC & -0.2869 & -0.4209 & -0.1799 & -0.0251 & 1 & & \\
\hline SPEC & -0.1967 & -0.1063 & -0.2292 & 0.8848 & -0.0026 & 1 & \\
\hline VART & -0.0609 & 0.0737 & -0.3140 & 0.4045 & -0.0596 & 0.3877 & 1 \\
\hline TL & -0.3306 & -0.0729 & -0.2604 & -0.1130 & 0.4431 & -0.0113 & -0.1538 \\
\hline TE & 0.1089 & -0.1603 & -0.3317 & 0.4066 & 0.1071 & 0.4376 & 0.3044 \\
\hline INV & 0.2494 & 0.1446 & -0.0193 & 0.0071 & -0.2906 & -0.0454 & -0.0408 \\
\hline LTA & -0.0942 & -0.2096 & 0.1763 & -0.7292 & 0.3499 & -0.6137 & -0.5111 \\
\hline NONII & 0.3454 & -0.6409 & 0.0284 & -0.1649 & 0.1688 & -0.1069 & -0.2043 \\
\hline GDP & -0.0070 & 0.2047 & 0.1210 & 0.0155 & -0.1152 & -0.0426 & -0.2805 \\
\hline INTEXP & -0.0534 & -0.0549 & -0.1660 & 0.2341 & -0.0998 & 0.2610 & 0.5009 \\
\hline
\end{tabular}

Table 2: Correlation matrix for all banks (next)

\begin{tabular}{|l|c|c|c|c|c|c|c|c|}
\hline & & TL & TE & INV & LTA & NONII & GDP & INTEXP \\
\hline TL & & 1 & & & & & \\
\hline TE & & 0.0767 & 1 & & & & \\
\hline INV & -0.1766 & -0.1304 & 1 & & & \\
\hline LTA & 0.4208 & -0.4808 & -0.0051 & 1 & & & \\
\hline NONII & 0.0146 & -0.0834 & 0.3006 & 0.4209 & 1 & & \\
\hline GDP & & 0.2052 & -0.2892 & 0.1590 & 0.1669 & 0.0502 & 1 & \\
\hline INTEXP & -0.0930 & 0.3269 & -0.4118 & -0.4203 & -0.2911 & -0.6884 & 1 \\
\hline
\end{tabular}


Table 3: Relationship between credit risks (measured by LLP) and lending structure concentration by GMM model in period 2011-2016

\begin{tabular}{|c|c|c|c|c|c|c|}
\hline & \multicolumn{6}{|c|}{$X^{*}$} \\
\hline & $\mathrm{RISKY}_{1}$ & $\mathrm{RISKY}_{2}$ & $\mathrm{RISKY}_{3}$ & $\mathrm{RISKY}_{4}$ & RISKY $_{5}$ & $\mathrm{RISKY}_{6}$ \\
\hline$X^{*}$ & $0.0424^{* *}$ & $0.0350^{* * *}$ & $0.1653^{* *}$ & $0.0434^{* *}$ & $0.0845^{*}$ & $0.1797^{\star * *}$ \\
\hline & $(0.020)$ & $(0.012)$ & $(0.073)$ & $(0.020)$ & $(0.047)$ & $(0.053)$ \\
\hline L.LLP & $-0.7764^{\star * *}$ & 0.1067 & $-0.6461^{*}$ & $-0.8999^{* *}$ & -0.2937 & -0.2262 \\
\hline & $(0.214)$ & $(0.247)$ & $(0.374)$ & $(0.365)$ & $(0.438)$ & $(0.201)$ \\
\hline NONII & & $1.8182^{* * *}$ & & -0.0520 & & $1.1162^{* *}$ \\
\hline & & $(0.440)$ & & $(1.275)$ & & $(0.529)$ \\
\hline INTEXP & $0.3484^{* * *}$ & $0.2111^{* * *}$ & $0.1594^{* * *}$ & $0.2324^{* *}$ & $0.1911^{* * *}$ & $0.2456^{* * *}$ \\
\hline & $(0.060)$ & $(0.034)$ & $(0.061)$ & $(0.114)$ & $(0.053)$ & $(0.028)$ \\
\hline TL & & & 0.0220 & 0.0294 & 0.0202 & $0.0518^{* *}$ \\
\hline & & & $(0.023)$ & $(0.022)$ & $(0.019)$ & $(0.021)$ \\
\hline L.TL & $0.0420 * *$ & & & & & \\
\hline & $(0.019)$ & & & & & \\
\hline TE & & & & & & \\
\hline INV & 0.0092 & & 0.0069 & & & \\
\hline & $(0.033)$ & & $(0.032)$ & & & \\
\hline GDP & & 0.0018 & & & & \\
\hline & & $(0.001)$ & & & & \\
\hline L.GDP & & & & & 0.0009 & \\
\hline & & & & & $(0.001)$ & \\
\hline Constant & -0.0266 & -0.0174 & -0.0040 & -0.0107 & -0.0130 & $-0.0356^{\star * *}$ \\
\hline & $(0.018)$ & $(0.012)$ & $(0.019)$ & $(0.013)$ & (0.009) & $(0.011)$ \\
\hline Observations & 40 & 40 & 40 & 40 & 40 & 40 \\
\hline $\begin{array}{l}\text { Number of } \\
\text { idbank }\end{array}$ & 8 & 8 & 8 & 8 & 8 & 8 \\
\hline Wald test & $\begin{array}{l}\text { Wald chi2(5) } \\
\quad=94.08 \\
\text { Prob > chi2 } \\
=0.000\end{array}$ & $\begin{array}{l}\text { Wald chi2(5) } \\
=347.15 \\
\text { Prob > chi2 } \\
=0.000\end{array}$ & $\begin{array}{l}\text { Wald chi2 }(5) \\
=493.23 \\
\text { Prob > chi2 } \\
=0.000\end{array}$ & $\begin{array}{l}\text { Wald chi2(5) } \\
\quad=28.38 \\
\text { Prob }>\text { chi2 } \\
=0.000\end{array}$ & $\begin{array}{l}\text { Wald chi2(5) } \\
\quad=54.50 \\
\text { Prob }>\text { chi2 } \\
=0.000\end{array}$ & $\begin{array}{l}\text { Wald chi2 }(5) \\
=1954.43 \\
\text { Prob }>\text { chi2 } \\
=0.000\end{array}$ \\
\hline $\begin{array}{l}\text { Endogenous } \\
\text { variables }\end{array}$ & $\begin{array}{l}\text { INTEXP, } \\
\operatorname{lag}(3 \text { 3) }\end{array}$ & $\begin{array}{l}\text { INTEXP, } \\
\operatorname{lag}(33)\end{array}$ & $\begin{array}{l}\text { INTEXP, } \\
\operatorname{lag}(33)\end{array}$ & $\begin{array}{l}\text { INTEXP, } \\
\operatorname{lag}(3 \text { 3) }\end{array}$ & $\begin{array}{l}\text { INTEXP, } \\
\operatorname{lag}(3 \text { 3) }\end{array}$ & $\begin{array}{l}\text { INTEXP, } \\
\operatorname{lag}(3 \text { 3) }\end{array}$ \\
\hline $\begin{array}{l}\text { Instrument } \\
\text { variables }\end{array}$ & L. RISKY & L. RISKY 2 & L. RISKY ${ }_{3}$ & L. $\mathrm{RISKY}_{4}$ & L.LTA & L. RISKY 6 \\
\hline $\begin{array}{l}\text { Number of } \\
\text { instruments }\end{array}$ & 8 & 8 & 8 & 8 & 8 & 8 \\
\hline
\end{tabular}




\begin{tabular}{|c|c|c|c|c|c|c|}
\hline & \multicolumn{6}{|c|}{$\mathbf{X}^{*}$} \\
\hline & RISKY $_{1}$ & $\mathrm{RISKY}_{2}$ & $\mathrm{RISKY}_{3}$ & RISKY $_{4}$ & RISKY $_{5}$ & RISKY $_{6}$ \\
\hline \multirow{4}{*}{$\begin{array}{l}\text { Arellano-Bond } \\
\text { test }\end{array}$} & \multicolumn{6}{|c|}{ Arellano-Bond test for $A R(1)$ in levels: } \\
\hline & $\begin{aligned} z= & 1.82 \operatorname{Pr}>z \\
= & 0.069\end{aligned}$ & $\begin{aligned} z= & 1.82 \mathrm{Pr}>\mathrm{z} \\
= & 0.069\end{aligned}$ & $\begin{aligned} z= & 1.37 \operatorname{Pr}>z \\
= & 0.170\end{aligned}$ & $\begin{aligned} z= & 1.67 \operatorname{Pr}>z \\
= & 0.095\end{aligned}$ & $\begin{aligned} z= & 1.57 \operatorname{Pr}>z \\
& =0.117\end{aligned}$ & $\begin{aligned} z= & 1.56 \operatorname{Pr}>z \\
= & 0.118\end{aligned}$ \\
\hline & \multicolumn{6}{|c|}{ Arellano-Bond test for $A R(2)$ in levels: } \\
\hline & $\begin{aligned} z= & 1.72 \operatorname{Pr}>z \\
= & 0.085\end{aligned}$ & $\begin{aligned} z= & 1.46 \operatorname{Pr}>z \\
= & 0.144\end{aligned}$ & $\begin{aligned} z= & 1.30 \operatorname{Pr}>z \\
= & 0.194\end{aligned}$ & $\begin{aligned} z= & 1.40 \operatorname{Pr}>z \\
= & 0.161\end{aligned}$ & $\begin{aligned} z= & 1.29 \operatorname{Pr}>z \\
= & 0.196\end{aligned}$ & $\begin{aligned} z= & 0.93 \operatorname{Pr}>z \\
= & 0.353\end{aligned}$ \\
\hline \multirow{2}{*}{$\begin{array}{l}\text { Sargan test of } \\
\text { overid }\end{array}$} & \multicolumn{6}{|c|}{ Sargan test of overid. restrictions: } \\
\hline & $\begin{array}{c}\text { chi2 }(2)=0.56 \\
\text { Prob }>\text { chi2 }= \\
0.757\end{array}$ & $\begin{array}{c}\text { chi2 }(2)=8.70 \\
\text { Prob }>\text { chi2 }= \\
0.013\end{array}$ & $\begin{array}{c}\text { chi2 }(2)=0.21 \\
\text { Prob }>\text { chi } 2= \\
0.902\end{array}$ & $\begin{array}{c}\text { chi } 2(2)=0.62 \\
\text { Prob }>\text { chi2 }= \\
0.735\end{array}$ & $\begin{array}{c}\text { chi2 }(2)=3.39 \\
\text { Prob }>\text { chi2 }= \\
0.184\end{array}$ & $\begin{array}{c}\operatorname{chi} 2(2)=1.02 \\
\text { Prob }>\text { chi2 }= \\
0.601\end{array}$ \\
\hline \multirow{2}{*}{$\begin{array}{l}\text { Hansen test of } \\
\text { overid }\end{array}$} & \multicolumn{6}{|c|}{ Hansen test of overid. restrictions: } \\
\hline & $\begin{array}{c}\text { chi2 }(2)=0.22 \\
\text { Prob }>\text { chi2 }= \\
0.897\end{array}$ & $\begin{array}{c}\text { chi } 2(2)=5.33 \\
\text { Prob }>\text { chi } 2= \\
0.070\end{array}$ & $\begin{array}{c}\text { chi2 }(2)=0.15 \\
\text { Prob }>\text { chi2 }= \\
0.926\end{array}$ & $\begin{array}{c}\text { chi2 }(2)=0.49 \\
\text { Prob }>\text { chi } 2= \\
0.782\end{array}$ & $\begin{array}{c}\text { chi } 2(2)=1.77 \\
\text { Prob }>\text { chi } 2= \\
0.413\end{array}$ & $\begin{array}{c}\text { chi2 }(2)=1.18 \\
\text { Prob }>\text { chi2 }= \\
0.555\end{array}$ \\
\hline \multirow{5}{*}{$\begin{array}{l}\text { Hansen test of } \\
\text { overid }\end{array}$} & \multicolumn{6}{|c|}{ Difference-in-Hansen tests of exogeneity of instrument subsets: } \\
\hline & \multicolumn{6}{|c|}{ Hansen test excluding group: } \\
\hline & $\begin{array}{c}\text { chi2 }(1)=0.04 \\
\text { Prob }>\text { chi2 }= \\
0.833\end{array}$ & $\begin{array}{c}\text { chi2 }(1)=3.28 \\
\text { Prob }>\text { chi2 }= \\
0.070\end{array}$ & $\begin{array}{c}\text { chi2 }(1)=0.10 \\
\text { Prob }>\text { chi2 }= \\
0.753\end{array}$ & $\begin{array}{c}\text { chi } 2(1)=0.32 \\
\text { Prob }>\text { chi2 }= \\
0.572\end{array}$ & $\begin{array}{c}\text { chi2 }(1)=0.03 \\
\text { Prob }>\text { chi2 }= \\
0.868\end{array}$ & $\begin{array}{c}\text { chi } 2(1)=0.12 \\
\text { Prob }>\text { chi } 2= \\
0.731\end{array}$ \\
\hline & \multicolumn{6}{|c|}{ Difference (null $\mathrm{H}=$ exogenous): } \\
\hline & $\begin{array}{c}\text { chi2 }(1)=0.17 \\
\text { Prob }>\text { chi2 }= \\
0.677\end{array}$ & $\begin{array}{c}\text { chi2 }(1)=2.05 \\
\text { Prob }>\text { chi2 }= \\
0.152\end{array}$ & $\begin{array}{c}\text { chi2 }(1)=0.05 \\
\text { Prob }>\text { chi2 }= \\
0.815\end{array}$ & $\begin{array}{c}\text { chi } 2(1)=0.17 \\
\text { Prob }>\text { chi2 }= \\
0.678\end{array}$ & $\begin{array}{c}\text { chi2 }(1)=1.74 \\
\text { Prob }>\text { chi2 }= \\
0.187\end{array}$ & $\begin{array}{c}\operatorname{chi} 2(1)=1.06 \\
\text { Prob }>\text { chi2 }= \\
0.304\end{array}$ \\
\hline
\end{tabular}

Table 3: Relationship between credit risks (measured by LLP) and lending structure concentration by GMM model in period 2011-2016 9 (next)

\begin{tabular}{|c|c|c|c|c|c|c|}
\hline & RISKY $_{7}$ & RISKY $_{8}$ & RISKY $_{9}$ & LCC & SPEC & VART \\
\hline \multirow[t]{2}{*}{$X^{*}$} & $-0.0395^{\star}$ & $-0.2281^{* *}$ & $-0.0208^{* * *}$ & $0.0425^{*}$ & $-0.0245^{\star *}$ & $-0.0577^{* * *}$ \\
\hline & $(0.021)$ & $(0.114)$ & $(0.006)$ & $(0.025)$ & $(0.010)$ & $(0.020)$ \\
\hline \multirow[t]{2}{*}{ L.LLP } & 0.0589 & -0.1469 & $-0.4728^{* *}$ & -0.4386 & $-0.4890^{* *}$ & 0.3041 \\
\hline & $(0.359)$ & $(0.205)$ & $(0.199)$ & $(0.288)$ & $(0.243)$ & $(0.237)$ \\
\hline \multirow[t]{2}{*}{ NONII } & & $0.9085^{* * *}$ & 0.6234 & 0.0566 & 1.0053 & $1.8186^{* * *}$ \\
\hline & & $(0.281)$ & $(0.519)$ & $(0.691)$ & $(0.612)$ & $(0.363)$ \\
\hline \multirow[t]{2}{*}{ INTEXP } & $0.3562^{* * *}$ & $0.2029^{* * *}$ & $0.2008^{* * *}$ & $0.2468^{* * *}$ & $0.2827^{* * *}$ & $0.4364^{* * *}$ \\
\hline & $(0.114)$ & $(0.060)$ & $(0.056)$ & $(0.057)$ & $(0.056)$ & $(0.085)$ \\
\hline \multirow[t]{2}{*}{$T \mathrm{TL}$} & & & $0.0244^{*}$ & & $0.0350^{*}$ & \\
\hline & & & $(0.014)$ & & $(0.021)$ & \\
\hline \multicolumn{7}{|l|}{ L.TL } \\
\hline \multirow[t]{2}{*}{ TE } & $-0.2856^{* *}$ & & & & & \\
\hline & $(0.137)$ & & & & & \\
\hline
\end{tabular}




\begin{tabular}{|c|c|c|c|c|c|c|}
\hline & RISKY $_{7}$ & RISKY $_{8}$ & RISKY $_{9}$ & LCC & SPEC & VART \\
\hline \multicolumn{7}{|l|}{ INV } \\
\hline \multirow{2}{*}{ GDP } & 0.0025 & & & 0.0014 & & $0.0061^{* * *}$ \\
\hline & $(0.003)$ & & & $(0.002)$ & & $(0.002)$ \\
\hline \multirow[t]{2}{*}{ L.GDP } & & $0.0013^{*}$ & & & & \\
\hline & & $(0.001)$ & & & & \\
\hline \multirow[t]{2}{*}{ Constant } & 0.0097 & -0.0021 & -0.0000 & -0.0412 & -0.0104 & $-0.0505^{\star \star *}$ \\
\hline & $(0.021)$ & $(0.009)$ & $(0.007)$ & $(0.033)$ & $(0.011)$ & $(0.017)$ \\
\hline Observations & 40 & 40 & 40 & 40 & 40 & 40 \\
\hline Number of idbank & 8 & 8 & 8 & 8 & 8 & 8 \\
\hline Wald test & $\begin{array}{c}\text { Wald chi2 }(5)= \\
24.46 \\
\text { Prob }>\text { chi2 }= \\
0.000\end{array}$ & $\begin{array}{c}\text { Wald chi2 }(5)= \\
492.59 \\
\text { Prob }>\text { chi2 }= \\
0.000\end{array}$ & $\begin{array}{c}\text { Wald chi2(5) }= \\
36.78 \\
\text { Prob }>\text { chi2 }= \\
0.000\end{array}$ & $\begin{array}{c}\text { Wald chi2(5) }= \\
56.27 \\
\text { Prob }>\text { chi2 }= \\
0.000\end{array}$ & $\begin{array}{c}\text { Wald chi2(5) }= \\
33.70 \\
\text { Prob }>\text { chi2 }= \\
0.000\end{array}$ & $\begin{array}{c}\text { Wald chi2 }(5)= \\
127.52 \\
\text { Prob }>\text { chi2 }= \\
0.000\end{array}$ \\
\hline $\begin{array}{l}\text { Endogenous } \\
\text { variables }\end{array}$ & $\begin{array}{c}\text { INTEXP, } \operatorname{lag}(3 \\
3)\end{array}$ & $\begin{array}{c}\text { INTEXP, } \operatorname{lag}(3 \\
3)\end{array}$ & $\begin{array}{c}\text { INTEXP, } \operatorname{lag}(3 \\
3)\end{array}$ & $\begin{array}{c}\text { INTEXP, } \operatorname{lag}(3 \\
3)\end{array}$ & $\begin{array}{c}\text { INTEXP, } \operatorname{lag}(3 \\
3)\end{array}$ & $\begin{array}{c}\text { INTEXP, } \operatorname{lag}(3 \\
3)\end{array}$ \\
\hline Instrument variables & L.ROA & L. RISKY 8 & L. RISKY & L.LCC & L.SPEC & L.VART \\
\hline $\begin{array}{l}\text { Number of } \\
\text { instruments }\end{array}$ & 8 & 8 & 8 & 8 & 8 & 8 \\
\hline \multirow[t]{4}{*}{ Arellano-Bond test } & \multicolumn{6}{|c|}{ Arellano-Bond test for AR(1) in levels: } \\
\hline & $\begin{aligned} z= & 1.81 \operatorname{Pr}>z \\
= & 0.071\end{aligned}$ & $\begin{aligned} z= & 2.43 \operatorname{Pr}>z \\
& =0.015\end{aligned}$ & $\begin{aligned} z= & 1.96 \operatorname{Pr}>z \\
& =0.050\end{aligned}$ & $\begin{aligned} z= & 1.53 \operatorname{Pr}>z \\
& =0.126\end{aligned}$ & $\begin{aligned} z= & 1.79 \operatorname{Pr}>z \\
& =0.074\end{aligned}$ & $\begin{aligned} z= & 1.96 \operatorname{Pr}>z \\
& =0.050\end{aligned}$ \\
\hline & \multicolumn{6}{|c|}{ Arellano-Bond test for AR(2) in levels: } \\
\hline & $\begin{aligned} z= & 1.67 \operatorname{Pr}>z \\
= & 0.096\end{aligned}$ & $\begin{aligned} z= & 1.68 \operatorname{Pr}>z \\
& =0.093\end{aligned}$ & $\begin{aligned} z= & 1.45 \operatorname{Pr}>z \\
& =0.147\end{aligned}$ & $\begin{aligned} z= & 1.39 \operatorname{Pr}>z \\
& =0.163\end{aligned}$ & $\begin{aligned} z= & 1.57 \operatorname{Pr}>z \\
& =0.117\end{aligned}$ & $\begin{aligned} z= & 0.35 \operatorname{Pr}>z \\
& =0.727\end{aligned}$ \\
\hline \multirow{2}{*}{$\begin{array}{l}\text { Sargan test of } \\
\text { overid }\end{array}$} & \multicolumn{6}{|c|}{ Sargan test of overid. restrictions: } \\
\hline & $\begin{array}{c}\text { chi2 } 2(2)=0.90 \\
\text { Prob }>\text { chi2 }= \\
0.639\end{array}$ & $\begin{array}{c}\text { chi2 }(2)=4.88 \\
\text { Prob }>\text { chi2 }= \\
0.087\end{array}$ & $\begin{array}{c}\text { chi2 } 2(2)=1.18 \\
\text { Prob }>\text { chi2 }= \\
0.555\end{array}$ & $\begin{array}{c}\text { chi2 } 2(2)=3.22 \\
\text { Prob }>\text { chi2 }= \\
0.20\end{array}$ & $\begin{array}{c}\text { chi2 }(2)=0.86 \\
\text { Prob }>\text { chi2 }= \\
0.650\end{array}$ & $\begin{array}{c}\text { chi2 } 2(2)=0.89 \\
\text { Prob }>\text { chi2 }= \\
0.642\end{array}$ \\
\hline \multirow{2}{*}{$\begin{array}{l}\text { Hansen test of } \\
\text { overid }\end{array}$} & \multicolumn{6}{|c|}{ Hansen test of overid. restrictions: } \\
\hline & $\begin{array}{c}\text { chi2 }(2)=0.18 \\
\text { Prob }>\text { chi2 }= \\
0.915\end{array}$ & $\begin{array}{c}\text { chi } 2(2)=2.69 \\
\text { Prob }>\text { chi2 }= \\
0.261\end{array}$ & $\begin{array}{c}\text { chi2 } 2(2)=1.23 \\
\text { Prob }>\text { chi2 }= \\
0.539\end{array}$ & $\begin{array}{c}\text { chi } 2(2)=2.18 \\
\text { Prob }>\text { chi2 }= \\
0.336\end{array}$ & $\begin{array}{c}\text { chi2 } 2(2)=0.96 \\
\text { Prob }>\text { chi2 }= \\
0.619\end{array}$ & $\begin{array}{c}\text { chi2 }(2)=0.40 \\
\text { Prob }>\text { chi2 }= \\
0.817\end{array}$ \\
\hline \multirow{5}{*}{$\begin{array}{l}\text { Hansen test of } \\
\text { overid }\end{array}$} & \multicolumn{6}{|c|}{ Difference-in-Hansen tests of exogeneity of instrument subsets: } \\
\hline & \multicolumn{6}{|c|}{ Hansen test excluding group: } \\
\hline & $\begin{array}{c}\text { chi2 }(1)=0.05 \\
\text { Prob }>\text { chi2 }= \\
0.820\end{array}$ & $\begin{array}{c}\text { chi2 }(1)=2.33 \\
\text { Prob }>\text { chi2 }= \\
0.127\end{array}$ & $\begin{array}{c}\text { chi2 } 2(1)=0.34 \\
\text { Prob }>\text { chi2 }= \\
0.559\end{array}$ & $\begin{array}{c}\text { chi2 }(1)=1.24 \\
\text { Prob }>\text { chi2 }= \\
0.265\end{array}$ & $\begin{array}{c}\text { chi2 } 2(1)=0.20 \\
\text { Prob }>\text { chi2 }= \\
0.652\end{array}$ & $\begin{array}{c}\text { chi2 }(1)=0.29 \\
\text { Prob }>\text { chi2 }= \\
0.588\end{array}$ \\
\hline & \multicolumn{6}{|c|}{ Difference (null $\mathrm{H}=$ exogenous): } \\
\hline & $\begin{array}{c}\text { chi2 }(1)=0.13 \\
\text { Prob }>\text { chi2 }= \\
0.723\end{array}$ & $\begin{array}{c}\text { chi2 }(1)=0.36 \\
\text { Prob }>\text { chi2 }= \\
0.549\end{array}$ & $\begin{array}{c}\text { chi2 } 2(1)=0.89 \\
\text { Prob }>\text { chi2 }= \\
0.345\end{array}$ & $\begin{array}{c}\text { chi2 }(1)=0.94 \\
\text { Prob }>\text { chi2 }= \\
0.333\end{array}$ & $\begin{array}{c}\text { chi2 } 2(1)=0.75 \\
\text { Prob }>\text { chi2 }= \\
0.385\end{array}$ & $\begin{array}{c}\text { chi } 2(1)=0.11 \\
\text { Prob }>\text { chi2 }= \\
0.740\end{array}$ \\
\hline
\end{tabular}


Table 4: Relationship between credit risks (measured by STDNPL) and lending structure concentration by GMM model in period 2011-2016

\begin{tabular}{|c|c|c|c|c|c|c|}
\hline & \multicolumn{6}{|c|}{$\mathbf{X}^{*}$} \\
\hline & RISKY & RISKY & $\mathrm{RISKY}_{3}$ & $\mathrm{RISKY}_{4}$ & RISKY $_{5}$ & RISKY $_{6}$ \\
\hline \multirow[t]{2}{*}{$X^{*}$} & $-0.0677^{* *}$ & $0.1030^{* * *}$ & $-0.9362^{* *}$ & $-0.0032^{* *}$ & $0.0149^{*}$ & 0.0571 \\
\hline & $(0.034)$ & $(0.022)$ & $(0.405)$ & $(0.001)$ & $(0.008)$ & $(0.053)$ \\
\hline \multirow[t]{2}{*}{ L.STDNPL } & $0.9932^{* * *}$ & $1.0009^{* * *}$ & $1.1103^{* * *}$ & $1.0014^{* \star *}$ & $1.0027^{* \star *}$ & $1.0080^{* * *}$ \\
\hline & $(0.009)$ & $(0.012)$ & $(0.091)$ & $(0.006)$ & $(0.002)$ & $(0.014)$ \\
\hline \multirow[t]{2}{*}{ NONII } & 3.0419 & 2.1642 & & & $-1.2299^{* * *}$ & \\
\hline & (1.959) & $(1.338)$ & & & $(0.265)$ & \\
\hline \multirow[t]{2}{*}{ INTEXP } & & & & & & $0.1445^{*}$ \\
\hline & & & & & & $(0.088)$ \\
\hline \multirow[t]{2}{*}{ LTA } & & & $-0.0254^{\star *}$ & & & \\
\hline & & & $(0.010)$ & & & \\
\hline \multirow[t]{2}{*}{ TL } & $-0.0123^{* * *}$ & $-0.0443^{*}$ & 0.0263 & $-0.0074^{\star * *}$ & & \\
\hline & $(0.005)$ & $(0.026)$ & $(0.106)$ & $(0.002)$ & & \\
\hline \multirow[t]{2}{*}{ INV } & -0.0386 & & & & & -0.0233 \\
\hline & $(0.025)$ & & & & & $(0.022)$ \\
\hline \multirow[t]{2}{*}{$\mathrm{TE}$} & & $-0.1391^{*}$ & & & & $-0.1393^{* *}$ \\
\hline & & $(0.083)$ & & & & $(0.058)$ \\
\hline \multirow[t]{2}{*}{ GDP } & & & -0.0018 & & & \\
\hline & & & $(0.002)$ & & & \\
\hline \multirow[t]{2}{*}{ Constant } & $0.0198^{* * *}$ & $0.0254^{*}$ & $0.3138^{\star * \star}$ & $0.0055^{\star * \star}$ & $0.0046^{\star \star *}$ & 0.0062 \\
\hline & $(0.005)$ & $(0.014)$ & $(0.087)$ & $(0.001)$ & $(0.001)$ & $(0.006)$ \\
\hline Observations & 40 & 40 & 40 & 40 & 40 & 40 \\
\hline $\begin{array}{l}\text { Number of } \\
\text { idbank }\end{array}$ & 8 & 8 & 8 & 8 & 8 & 8 \\
\hline Wald test & $\begin{array}{c}\text { Wald } \\
\text { chi2 } 2(5)= \\
1.41 \mathrm{e}+06 \\
\text { Prob }>\text { chi2 } \\
=\quad 0.000\end{array}$ & $\begin{array}{l}\text { Wald chi2(5) } \\
=46071.13 \\
\text { Prob }>\text { chi2 } \\
=\quad 0.000\end{array}$ & $\begin{array}{c}\text { Wald chi2(5) }= \\
1014.85 \\
\text { Prob }>\text { chi } 2= \\
0.000\end{array}$ & $\begin{array}{c}\text { Wald chi2(3) }= \\
70615.73 \\
\text { Prob }>\text { chi2 }= \\
0.000\end{array}$ & $\begin{array}{c}\text { Wald chi2(3) }= \\
3.68 \mathrm{e}+06 \\
\text { Prob }>\text { chi2 }= \\
0.000\end{array}$ & $\begin{array}{c}\text { Wald chi2(5) }= \\
268592.10 \\
\text { Prob }>\text { chi2 }= \\
0.000\end{array}$ \\
\hline $\begin{array}{l}\text { Endogenous } \\
\text { variables }\end{array}$ & 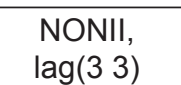 & TL, lag(3 3) & TL, lag(3 3) & TL, lag(3 3 & NONII, lag(3 3) & INTEXP, lag(3 3) \\
\hline $\begin{array}{l}\text { Instrument } \\
\text { variables }\end{array}$ & LTA & LTA & L.GDP & L.RISKY4 & L.LTA & L.INTEXP \\
\hline $\begin{array}{l}\text { Number of } \\
\text { instruments }\end{array}$ & 8 & 8 & 8 & 8 & 8 & 8 \\
\hline \multirow{4}{*}{$\begin{array}{l}\text { Arellano-Bond } \\
\text { test }\end{array}$} & \multicolumn{6}{|c|}{ Arellano-Bond test for AR(1) in levels: } \\
\hline & $\begin{array}{c}z=0.89 \\
\operatorname{Pr}>z= \\
0.376\end{array}$ & $\begin{array}{l}z=1.51 \operatorname{Pr} \\
>z=0.131\end{array}$ & $\begin{aligned} z= & 1.62 \operatorname{Pr}>z \\
= & 0.106\end{aligned}$ & $\begin{aligned} z= & 0.47 \operatorname{Pr}>z \\
= & 0.635\end{aligned}$ & $\begin{array}{c}z=0.79 P r> \\
z=0.432\end{array}$ & $\begin{aligned} z= & 0.79 \operatorname{Pr}>z \\
= & 0.431\end{aligned}$ \\
\hline & \multicolumn{6}{|c|}{ Arellano-Bond test for AR(2) in levels: } \\
\hline & $\begin{array}{c}z=0.80 \\
\operatorname{Pr}>z= \\
0.425\end{array}$ & $\begin{array}{l}z=1.21 \mathrm{Pr} \\
>\mathrm{z}=0.228\end{array}$ & $\begin{aligned} z= & 1.62 \operatorname{Pr}>z \\
= & 0.106\end{aligned}$ & $\begin{aligned} z= & 1.48 \mathrm{Pr}>\mathrm{z} \\
= & 0.138\end{aligned}$ & $\begin{array}{c}z=0.18 \operatorname{Pr}> \\
z=0.860\end{array}$ & $\begin{aligned} z= & 0.42 \operatorname{Pr}>z \\
= & 0.671\end{aligned}$ \\
\hline
\end{tabular}




\begin{tabular}{|c|c|c|c|c|c|c|}
\hline & \multicolumn{6}{|c|}{$\mathbf{X}^{*}$} \\
\hline & RISKY $_{1}$ & $\mathrm{RISKY}_{2}$ & RISKY $_{3}$ & RISKY $_{4}$ & $\mathrm{RISKY}_{5}$ & RISKY $_{6}$ \\
\hline \multirow{2}{*}{$\begin{array}{l}\text { Sargan test of } \\
\text { overid }\end{array}$} & \multicolumn{6}{|c|}{ Sargan test of overid. restrictions: } \\
\hline & $\begin{array}{c}\text { chi2 }(2)= \\
0.07 \text { Prob } \\
>\text { chi2 }= \\
0.963\end{array}$ & $\begin{array}{c}\text { chi2 }(2)= \\
0.50 \text { Prob }> \\
\text { chi2 }=0.779\end{array}$ & $\begin{array}{c}\text { chi2(2) }=2.59 \\
\text { Prob }>\text { chi2 }= \\
0.273\end{array}$ & $\begin{array}{c}\text { chi2 }(4)=1.65 \\
\text { Prob }>\text { chi2 }= \\
0.800\end{array}$ & $\begin{array}{c}\text { chi2(4) = } \\
1.23 \text { Prob > } \\
\text { chi2 }=0.873\end{array}$ & $\begin{array}{c}\text { chi2(2) }=1.91 \\
\text { Prob }>\text { chi2 }= \\
0.385\end{array}$ \\
\hline \multirow{7}{*}{$\begin{array}{l}\text { Hansen test of } \\
\text { overid }\end{array}$} & \multicolumn{6}{|c|}{ Hansen test of overid. restrictions: } \\
\hline & $\begin{array}{c}\text { chi2 }(2)= \\
1.31 \text { Prob } \\
>\text { chi2 }= \\
0.519\end{array}$ & $\begin{array}{c}\text { chi2(2) = } \\
0.57 \text { Prob > } \\
\text { chi2 }=0.752\end{array}$ & $\begin{array}{c}\text { chi2(2) }=1.81 \\
\text { Prob }>\text { chi2 }= \\
0.404\end{array}$ & $\begin{array}{c}\text { chi2 }(4)=1.58 \\
\text { Prob }>\text { chi2 }= \\
0.813\end{array}$ & $\begin{array}{c}\text { chi2(4) = } \\
3.86 \text { Prob > } \\
\text { chi2 }=0.425\end{array}$ & $\begin{array}{c}\text { chi2 }(2)=1.87 \\
\text { Prob }>\text { chi2 }= \\
0.392\end{array}$ \\
\hline & \multicolumn{6}{|c|}{ Difference-in-Hansen tests of exogeneity of instrument subsets: } \\
\hline & \multicolumn{6}{|c|}{ Hansen test excluding group: } \\
\hline & $\begin{array}{c}\text { chi2 }(1)= \\
0.05 \text { Prob } \\
>\text { chi2 }= \\
0.830\end{array}$ & $\begin{array}{c}\text { chi2 }(1)= \\
0.00 \text { Prob > } \\
\text { chi2 }=0.960\end{array}$ & $\begin{array}{c}\text { chi2 }(1)=0.16 \\
\text { Prob }>\text { chi2 }= \\
0.685\end{array}$ & $\begin{array}{c}\text { chi2 }(3)=1.57 \\
\text { Prob }>\text { chi2 }= \\
0.667\end{array}$ & $\begin{array}{c}\text { chi2 }(3)= \\
1.16 \text { Prob > } \\
\text { chi2 }=0.762\end{array}$ & $\begin{array}{c}\text { chi2(1) = } 1.87 \\
\text { Prob }>\text { chi2 }= \\
0.171\end{array}$ \\
\hline & \multicolumn{6}{|c|}{ Difference (null $\mathrm{H}=$ exogenous): } \\
\hline & $\begin{array}{c}\text { chi2 }(1)= \\
1.26 \text { Prob } \\
>\text { chi2 }= \\
0.261\end{array}$ & $\begin{array}{c}\text { chi2 }(1)= \\
0.57 \text { Prob > } \\
\text { chi2 }=0.451\end{array}$ & $\begin{array}{c}\text { chi2 }(1)=1.65 \\
\text { Prob }>\text { chi2 }= \\
0.199\end{array}$ & $\begin{array}{c}\text { chi2 }(1)=0.01 \\
\text { Prob }>\text { chi2 }= \\
0.929\end{array}$ & $\begin{array}{c}\text { chi2(1) = } \\
2.70 \text { Prob > } \\
\text { chi2 }=0.100\end{array}$ & $\begin{array}{c}\text { chi2(1) }=0.00 \\
\text { Prob }>\text { chi } 2= \\
0.977\end{array}$ \\
\hline
\end{tabular}

Table 4: Relationship between credit risks (measured by STDNPL) and lending structure concentration by GMM model in period 2011-2016 (next)

\begin{tabular}{|c|c|c|c|c|c|c|}
\hline & \multicolumn{6}{|c|}{$X^{*}$} \\
\hline & RISKY $_{7}$ & $\mathrm{RISKY}_{8}$ & RISKY $_{9}$ & LCC & SPEC & VART \\
\hline \multirow[t]{2}{*}{$X^{*}$} & $0.0381^{* *}$ & $-0.1375^{\star *}$ & -0.0014 & $-0.0125^{* *}$ & $-0.0358^{* *}$ & $-0.0132^{* *}$ \\
\hline & $(0.017)$ & $(0.062)$ & $(0.005)$ & $(0.006)$ & $(0.014)$ & $(0.006)$ \\
\hline \multirow[t]{2}{*}{ L.STDNPL } & $0.9970^{* * *}$ & $0.9911^{* * *}$ & $1.0024^{* * *}$ & $1.0021^{\text {***}}$ & $1.0846^{* * *}$ & $1.0066^{* * *}$ \\
\hline & $(0.004)$ & $(0.013)$ & $(0.007)$ & $(0.004)$ & $(0.063)$ & $(0.004)$ \\
\hline \multirow[t]{2}{*}{ NONII } & $1.5954^{*}$ & & & -0.2594 & & \\
\hline & $(0.853)$ & & & $(0.265)$ & & \\
\hline \multirow[t]{2}{*}{ L.NONII } & & & & & $-7.9578^{*}$ & \\
\hline & & & & & $(4.678)$ & \\
\hline \multirow[t]{2}{*}{ INTEXP } & $0.1069^{* * *}$ & & $0.0789^{* *}$ & $0.0832^{* * *}$ & -0.1261 & $0.1683^{* * *}$ \\
\hline & $(0.041)$ & & $(0.038)$ & $(0.027)$ & $(0.106)$ & $(0.051)$ \\
\hline \multirow[t]{2}{*}{ LTA } & & 0.0004 & 0.0004 & $0.0016^{* * *}$ & & \\
\hline & & $(0.001)$ & $(0.001)$ & $(0.000)$ & & \\
\hline \multirow[t]{2}{*}{ TL } & & -0.0055 & & & & \\
\hline & & $(0.015)$ & & & & \\
\hline \multirow[t]{2}{*}{ INV } & $-0.0300^{*}$ & 0.0077 & $0.0078^{* *}$ & & & -0.0094 \\
\hline & $(0.016)$ & $(0.025)$ & $(0.004)$ & & & $(0.006)$ \\
\hline \multirow[t]{2}{*}{ GDP } & & & & & -0.0012 & $0.0017^{* *}$ \\
\hline & & & & & $(0.001)$ & $(0.001)$ \\
\hline \multirow[t]{2}{*}{ Constant } & $-0.0094^{* *}$ & 0.0008 & -0.0091 & -0.0106 & $0.0390^{* *}$ & $-0.0155^{\text {** }}$ \\
\hline & $(0.005)$ & $(0.011)$ & $(0.015)$ & $(0.008)$ & $(0.017)$ & $(0.006)$ \\
\hline
\end{tabular}




\begin{tabular}{|c|c|c|c|c|c|c|}
\hline & \multicolumn{6}{|c|}{$\mathbf{X}^{*}$} \\
\hline & RISKY $_{7}$ & RISKY $_{8}$ & RISKY & LCC & SPEC & VART \\
\hline Observations & 40 & 40 & 40 & 40 & 40 & 40 \\
\hline $\begin{array}{l}\text { Number of } \\
\text { idbank }\end{array}$ & 8 & 8 & 8 & 8 & 8 & 8 \\
\hline Wald test & $\begin{array}{l}\text { Wald chi2(5) } \\
=29933.52 \\
\text { Prob }>\text { chi2 } \\
=0.000\end{array}$ & $\begin{array}{c}\text { Wald chi2 } 2(5)= \\
2.08 \mathrm{e}+06 \\
\text { Prob }>\text { chi2 }= \\
0.000\end{array}$ & $\begin{array}{c}\text { Wald chi2 }(5)= \\
310084.55 \\
\text { Prob }>\text { chi2 }= \\
0.000\end{array}$ & $\begin{array}{c}\text { Wald chi2 }(5)= \\
32495.57 \\
\text { Prob }>\text { chi } 2= \\
0.000\end{array}$ & $\begin{array}{c}\text { Wald chi2 } 2(5)= \\
415110.83 \\
\text { Prob }>\text { chi2 }= \\
0.000\end{array}$ & $\begin{array}{c}\text { Wald chi2(5) }= \\
29933.52 \\
\text { Prob }>\text { chi2 }=0.000\end{array}$ \\
\hline $\begin{array}{l}\text { Endogenous } \\
\text { variables }\end{array}$ & INV, lag(3 3) & $\begin{array}{c}\text { INTEXP, lag(3 } \\
3)\end{array}$ & $\begin{array}{l}\text { INTEXP, } \operatorname{lag}(3 \\
3)\end{array}$ & $\begin{array}{l}\text { INTEXP, } \operatorname{lag}(3 \\
3)\end{array}$ & $\begin{array}{c}\text { INTEXP, lag(3 } \\
3)\end{array}$ & INV, lag(3 3) \\
\hline $\begin{array}{l}\text { Instrument } \\
\text { variables }\end{array}$ & L.RISKY8 & L.GDP & GDP & $\mathrm{ROA}$ & L.GDP & L.RISKY8 \\
\hline $\begin{array}{l}\text { Number of } \\
\text { instruments }\end{array}$ & 8 & 8 & 8 & 8 & 8 & 8 \\
\hline \multirow{4}{*}{$\begin{array}{l}\text { Arellano-Bond } \\
\text { test }\end{array}$} & \multicolumn{6}{|c|}{ Arellano-Bond test for AR(1) in levels: } \\
\hline & $\begin{array}{c}z=0.16 \operatorname{Pr}> \\
z=0.874\end{array}$ & $\begin{aligned} z= & 0.34 \operatorname{Pr}>z \\
& =0.736\end{aligned}$ & $\begin{aligned} z= & 0.16 \operatorname{Pr}>z \\
& =0.872\end{aligned}$ & $\begin{aligned} z= & -0.23 \operatorname{Pr}>z \\
& =0.821\end{aligned}$ & $\begin{aligned} z= & 1.31 \operatorname{Pr}>z \\
& =0.189\end{aligned}$ & $\begin{array}{c}z=0.25 \operatorname{Pr}>z= \\
0.805\end{array}$ \\
\hline & \multicolumn{6}{|c|}{ Arellano-Bond test for AR(2) in levels: } \\
\hline & $\begin{array}{c}z=0.17 \mathrm{Pr}> \\
z=0.866\end{array}$ & $\begin{aligned} z= & 0.47 \operatorname{Pr}>z \\
& =0.639\end{aligned}$ & $\begin{aligned} z= & 0.96 \operatorname{Pr}>z \\
& =0.336\end{aligned}$ & $\begin{aligned} z= & 0.26 \operatorname{Pr}>z \\
& =0.796\end{aligned}$ & $\begin{aligned} z= & 1.28 \operatorname{Pr}>z \\
& =0.200\end{aligned}$ & $\begin{array}{c}z=0.92 \operatorname{Pr}>z= \\
0.359\end{array}$ \\
\hline \multirow{2}{*}{$\begin{array}{l}\text { Sargan test of } \\
\text { overid }\end{array}$} & \multicolumn{6}{|c|}{ Sargan test of overid. restrictions: } \\
\hline & $\begin{array}{c}\text { chi2 }(2)= \\
2.35 \text { Prob }> \\
\text { chi2 }=0.308\end{array}$ & $\begin{array}{c}\text { chi2 } 2(2)=1.53 \\
\text { Prob }>\text { chi } 2= \\
0.465\end{array}$ & $\begin{array}{c}\text { chi } 2(2)=4.04 \\
\text { Prob }>\text { chi2 }= \\
0.133\end{array}$ & $\begin{array}{c}\text { chi } 2(2)=2.83 \\
\text { Prob }>\text { chi2 }= \\
0.243\end{array}$ & $\begin{array}{c}\text { chi2 } 2(2)=1.16 \\
\text { Prob }>\text { chi2 }= \\
0.560\end{array}$ & $\begin{array}{c}\text { chi2 } 2(2)=2.55 \text { Prob } \\
>\text { chi } 2=0.280\end{array}$ \\
\hline \multirow{7}{*}{$\begin{array}{l}\text { Hansen test of } \\
\text { overid }\end{array}$} & \multicolumn{6}{|c|}{ Hansen test of overid. restrictions: } \\
\hline & $\begin{array}{c}\text { chi2 }(2)= \\
2.40 \text { Prob > } \\
\text { chi2 }=0.301\end{array}$ & $\begin{array}{c}\text { chi2 } 2(2)=1.46 \\
\text { Prob }>\text { chi2 }= \\
0.482\end{array}$ & $\begin{array}{c}\operatorname{chi} 2(2)=3.01 \\
\text { Prob }>\text { chi2 }= \\
0.222\end{array}$ & $\begin{array}{c}\text { chi2 }(2)=3.12 \\
\text { Prob }>\text { chi2 }= \\
0.211\end{array}$ & $\begin{array}{c}\text { chi2 } 2(2)=0.88 \\
\text { Prob }>\text { chi2 }= \\
0.643\end{array}$ & $\begin{array}{c}\text { chi2 } 2(2)=3.04 \text { Prob } \\
>\text { chi2 }=0.219\end{array}$ \\
\hline & \multicolumn{6}{|c|}{ Difference-in-Hansen tests of exogeneity of instrument subsets: } \\
\hline & \multicolumn{6}{|c|}{ Hansen test excluding group: } \\
\hline & $\begin{array}{c}\text { chi2 }(1)= \\
2.01 \text { Prob > } \\
\text { chi2 }=0.156\end{array}$ & $\begin{array}{c}\text { chi2 }(1)=1.46 \\
\text { Prob }>\text { chi2 }= \\
0.227\end{array}$ & $\begin{array}{c}\text { chi2 }(1)=2.76 \\
\text { Prob }>\text { chi2 }= \\
0.096\end{array}$ & $\begin{array}{c}\text { chi2 } 2(1)=2.81 \\
\text { Prob }>\text { chi2 }= \\
0.094\end{array}$ & $\begin{array}{c}\text { chi2 }(1)=0.04 \\
\text { Prob }>\text { chi2 }= \\
0.846\end{array}$ & $\begin{array}{c}\text { chi2 }(1)=3.04 \text { Prob } \\
>\text { chi2 }=0.081\end{array}$ \\
\hline & \multicolumn{6}{|c|}{ Difference (null $\mathrm{H}=$ exogenous): } \\
\hline & $\begin{array}{c}\text { chi2 }(1)= \\
0.39 \text { Prob > } \\
\text { chi2 }=0.535\end{array}$ & $\begin{array}{c}\text { chi } 2(1)=0.00 \\
\text { Prob }>\text { chi } 2= \\
0.987\end{array}$ & $\begin{array}{c}\text { chi2 }(1)=0.24 \\
\text { Prob }>\text { chi2 }= \\
0.622\end{array}$ & $\begin{array}{c}\text { chi2 }(1)=0.31 \\
\text { Prob }>\text { chi2 }= \\
0.580\end{array}$ & $\begin{array}{c}\text { chi2 }(1)=0.85 \\
\text { Prob }>\text { chi2 }= \\
0.358\end{array}$ & $\begin{array}{c}\text { chi } 2(1)=0.00 \text { Prob } \\
>\text { chi2 }=0.968\end{array}$ \\
\hline
\end{tabular}

For this sample, estimations were separately run for different independent variables (measured by RISKY1, RISKY2, RISKY3, RISKY4, RISKY5, RISKY6, RISKY7, RISKY8, RISKY9, SPEC, LCC, VART). It is found that most of the coefficients for the main independent variables focusing on two measures of credit risks are statistically significant.

It is evident that the impacts of loan portfolio concentration vary across economic sectors. It is interesting to observe that there are significant positive correlations between variables (RISKY2, RISKY5, respectively) with LLP and STDNPL. This provides strong evidence to argue that an increase in bank lending for the agriculture, electricity, gas and water contributes to the bank's exposures to credit risks. Meanwhile, the negative relationship of RISKY8 with both LLP and STDNPL is noted, suggesting that bank lending concentration into hotels and restaurants reduces credit risk. However, this result shows that variables RISKY1, RISKY3, RISKY4, RISKY7 and LCC have a negative correlation with STDNPL, but a positive correlation with LLP.

More importantly, it is also pointed out that the coefficient of SPEC and VART is negative and significant with LLP and STDNPL. That means an increase in bank lending concentration in certain economic sectors reduces 
credit risk. This is consistent with the findings of Acharya et al. (2006).

The finding from the lagged control variables (L.LLP and L.STDNPL) also shows that credit risk in the previous year impacts credit risk in the current year. In addition, the positive impact of non-interest income (measured by NONII) on STDNPL and LLP suggests that low levels of non-interest income are risk-reducing (Lepetit, Nys, Rous, \& Tarazi, 2008). Therefore, non-interest income may increase the volatility of banking income because lending operations need lower operating leverage than fee collection activities (DeYoung \& Roland, 2001).

The coefficient of INTEXP, STDNPL and LLP are positively significant in almost all models. This result suggests that credit-expanding strategies are considered less risky. However, this result is contradictory to the findings of Lepetit et al. (2008) and Stiroh (2004), suggesting that greater reliance on non-interest income is associated with higher risk and lower risk-adjusted profit. It implies that the banks play a key role in Vietnam's financial market. And it mainly focuses on mobilizing deposits and making loans.

Meanwhile, it is noted that there is a positive relationship between variables (RISKY2, RISKY4, RISKY6, RISKY7, RISKY 8 and RISKY9) and RSS, suggesting that a decrease in lending in agriculture, manufacturing, transport and communication, construction and real estate, hotels and restaurants, and other community reduces credit risk.

\section{Conclusion}

The study applies the GMM estimation method to assess the impact of concentrated loan structure on credit risks of Vietnamese commercial banks in the period from 2011 to 2016. The results show that commercial banks with a low level of concentration may cause greater credit risk.

Considering the impact of outstanding loans of each economic sector on credit risk, the research results suggest that Vietnam's commercial banks should expand credit for the hotel and restaurant sector. However, credit expansion should be considered with strict restrictions for economic sectors with a high requirement for capital, such as agriculture, electricity, gas and water. This evidence implies the importance of lending concentration in decreasing credit risk. The study also shows that boosting credit growth in economic sectors can reduce bad debts. This implies that the banks should revolve credit quickly by replacing old debts with new ones. However, the growth of outstanding loans is potentially risky, so the growth must rely on credit efficiency. As a result, when banks increase credit balance, they may increase the provision of credit risk.

From the results and policy implications mentioned above, the current paper equips bank managers with a good understanding of lending structure concentration and credit risk to deal with loan expansion and monitoring efficiency when determining credit risk exposure. Moreover, it also provides policymakers with better analyses, which can help them achieve the best possible credit policy transmission mechanism.

\section{References}

Abdul-Rahman, A., Sulaiman, A. A., \& Mohd Said, N. L. H. (2018). Does financing structure affects bank liquidity risk? PacificBasin Finance Journal, 52, 26-39. https://doi.org/10.1016/j. pacfin.2017.04.004

Acharya, V.V., Hasan, I., \& Saunders, A. (2006). Should Banks Be Diversified? Evidence from Individual Bank Loan Portfolios. The Journal of Business, 79(3), 1355-1412. https://doi. org/10.1086/500679

Arellano, M., \& Bover, O. (1995). Another look at the instrumental variable estimation of error-components models. Journal of Econometrics, 68(1), 29-51. https://doi.org/10.1016/03044076(94)01642-D

Bassett, W., Demiralp, S., \& Lloyd, N. (2020). Government support of banks and bank lending. Journal of Banking \& Finance, 112, 105177. https://doi.org/10.1016/j.jbankfin.2017.07.010

Batten, J., \& Vo, X. V. (2016). Bank risk shifting and diversification in an emerging market. Risk Management, 18. https://doi. org/10.1057/s41283-016-0008-2

Batten, J. A., \& Vo, X. V. (2015). Foreign ownership in emerging stock markets. Journal of Multinational Financial Management, 32-33, 15-24. https://doi.org/10.1016/j.mulfin.2015.05.001

Behr, A., Kamp, A., Memmel, C., \& Pfingsten, A. (2007). Diversification and the Banks' Risk-Return-Characteristics - Evidence from Loan Portfolios of German Banks. SSRN Electronic Journal. https://doi.org/10.2139/ssrn.906448

Berger, A. N., \& DeYoung, R. (1997). Problem loans and cost efficiency in commercial banks. Journal of Banking \& Finance, 21(6), 849-870. https://doi.org/10.1016/S0378-4266(97)00003-4

Berger, P. G., Minnis, M., \& Sutherland, A. (2017). Commercial lending concentration and bank expertise: Evidence from borrower financial statements. Journal of Accounting and Economics, 64(2), 253-277. https://doi.org/10.1016/j. jacceco.2017.06.005

Blasko, M., \& Sinkey, J. (2006). Bank Asset Structure, RealEstate Lending, and Risk-Taking. The Quarterly Review of Economics and Finance, 46, 53-81. https://doi.org/10.1006/ jfin.2000.030510.1016/j.qref.2004.11.002

Chen, Y., Wei, X., \& Zhang, L. (2013). A New Measurement of Sectoral Concentration of Credit Portfolios. Procedia Computer Science, 17, 1231-1240. https://doi.org/10.1016/j. procs.2013.05.157

Dao, B. T. T., \& Nguyen, D. P. (2020). Determinants of Profitability in Commercial Banks in Vietnam, Malaysia and Thailand. Journal of Asian Finance, Economics and Business, 7(4), 133143. https://doi.org/10.13106/jafeb.2020.vol7.no4.133 
DeYoung, R., \& Roland, K. P. (2001). Product Mix and Earnings Volatility at Commercial Banks: Evidence from a Degree of Total Leverage Model. Journal of Financial Intermediation, 10(1), 54-84. https://doi.org/10.1006/jfin.2000.0305

Diallo, B. (2017). Corporate Governance, Bank Concentration and Economic Growth. Emerging Markets Review, 32. https://doi. org/10.1016/j.ememar.2017.05.003

Diamond, D. W. (1984). Financial Intermediation and Delegated Monitoring. The Review of Economic Studies, 51(3), 393-414. https://doi.org/10.2307/2297430

Haq, M., \& Heaney, R. (2012). Factors determining European bank risk. Journal of International Financial Markets, Institutions and Money, 22(4), 696-718. https://doi.org/10.1016/j. intfin.2012.04.003

Kahn, C., \& Winton, A. (2004). Moral Hazard and Optimal Subsidiary Structure for Financial Institutions. The Journal of Finance, 59(6), 2531-2575. https://doi.org/10.1111/j.15406261.2004.00708.x

Kildegaard, A., \& Williams, P. (2002). Banks, systematic risk, and industrial concentration: theory and evidence. Journal of Economic Behavior \& Organization, 47(4), 345-358. https:// doi.org/10.1016/S0167-2681(01)00200-1

Lepetit, L., Nys, E., Rous, P., \& Tarazi, A. (2008). Bank income structure and risk: An empirical analysis of European banks. Journal of Banking \& Finance, 32(8), 1452-1467. https://doi. org/10.1016/j.jbankfin.2007.12.002

Luong, T. T. H., Nguyen, T. M., \& Nguyen, T. A. N. (2020). Rule of Law, Economic Growth and Shadow Economy in Transition Countries. Journal of Asian Finance, Economics and Business, 7(4), 145-154. https://doi.org/10.13106/jafeb.2020.vol7.no4.145

Markowitz, H. M. (1959). Portfolio Selection Efficient Diversification of Investments. New Haven, Connecticut: Yale University Press.

Nepp, A. N., Lavysh, A. A., Kuprina, T. V., \& Nikonov, O. I. (2012). Optimization of the Branch Structure of Bank's Loan Portfolio. IFAC Proceedings Volumes, 45(25), 246-250. https:// doi.org/10.3182/20120913-4-IT-4027.00055

Onyiriuba, L. (2016). Chapter 6 - Bank Marketing Paradox and Risks Management in Developing Economies. In L. Onyiriuba (Ed.), Bank Risk Management in Developing Economies (pp. 97-111): Academic Press.
Ozili, P. K., \& Outa, E. (2017). Bank loan loss provisions research: A review. Borsa Istanbul Review, 17(3), 144-163. https://doi. org/10.1016/j.bir.2017.05.001

Rahman, A. A. (2010). Financing structure and insolvency risk exposure of Islamic banks. Financial Markets and Portfolio Management, 24(4), 419-440. https://doi.org/10.1006/ jfin.2000.030510.1007/s11408-010-0142-x

Rossi, S. P. S., Schwaiger, M. S., \& Winkler, G. (2009). How loan portfolio diversification affects risk, efficiency and capitalization: A managerial behavior model for Austrian banks. Journal of Banking \& Finance, 33(12), 2218-2226. https://doi. org/10.1016/j.jbankfin.2009.05.022

Silva, T. C., Alexandre, M. d. S., \& Tabak, B. M. (2018). Bank lending and systemic risk: A financial-real sector network approach with feedback. Journal of Financial Stability, 38, 98118. https://doi.org/10.1016/j.jfs.2017.08.006

Stiroh, K. J. (2004). Diversification in Banking: Is Noninterest Income the Answer. Journal of money credit and banking, 36, 853-882. https://doi.org/10.1353/mcb.2004.0076

Tabak, B. M., Fazio, D. M., \& Cajueiro, D. O. (2011). The effects of loan portfolio concentration on Brazilian banks' return and risk. Journal of Banking \& Finance, 35(11), 3065-3076. https://doi. org/10.1016/j.jbankfin.2011.04.006

Tran, S. H., \& Nguyen, L. T. (2020). Financial Development, Business Cycle and Bank Risk in Southeast Asian Countries. Journal of Asian Finance, Economics and Business, 7(3), 127135. https://doi.org/10.13106/jafeb.2020.vol7.no3.127

van der Veer, K. J. M., \& Hoeberichts, M. M. (2016). The level effect of bank lending standards on business lending. Journal of Banking \& Finance, 66, 79-88. https://doi.org/10.1016/j. jbankfin.2016.01.003

Vietnamnet. (2017, 19/01/2017). Vietnam's economic challenges in 2017. Retrieved from https://english.vietnamnet.vn/fms/ business/171104/vietnam-s-economic-challenges-in-2017.html

Vo, X. V. (2015). Foreign ownership and stock return volatility Evidence from Vietnam. Journal of Multinational Financial Management, 30, 101-109. https://doi.org/10.1016/j. mulfin.2015.03.004

Vo, X. V. (2017). How does the stock market value bank diversification? Evidence from Vietnam. Finance Research Letters, 22, 101-104. https://doi.org/10.1016/j.frl.2017.06.005 